\title{
KNOTTED PROJECTIONS OF PLANAR GRAPHS
}

\author{
KOUKI TANIYAMA
}

(Communicated by Ronald J. Stern)

\begin{abstract}
In this paper we describe a regular projection of a planar graph that is achieved only by knotted embeddings in 3-space. We also give conditions under which any regular projection of such graphs can be achieved by unknotted embeddings.
\end{abstract}

\section{INTRODUCTION}

Throughout this paper we work in the piecewise-linear category. Let $G$ be a finite graph. We think of $G$ as a topological space in the usual way. A regular projection of $G$ is the image of a continuous map from $G$ to the 2-dimensional Euclidean space $R^{2}$ whose multiple points are only finitely many transversal double points away from the vertices of $G$. Suppose that $R^{2} \subset R^{3}$. If we give over/under information to each double point, then the regular projection represents a graph embedded in $R^{3}$ up to ambient isotopy.

A graph $G$ is called planar if there is an embedding of $G$ into $R^{2}$. An embedded graph $G$ in $R^{3}$ is called unknotted if $G$ is ambient isotopic to a graph in $R^{2} \subset R^{3}$. Thus $G$ is unknotted only if $G$ is planar. It is shown in [2] that if $G_{1}$ and $G_{2}$ are unknotted graphs and $G_{1}$ is homeomorphic to $G_{2}$, then $G_{1}$ is ambient isotopic to $G_{2}$ in $R^{3}$.

It is well known in Knot Theory that a regular projection of a disjoint union of circles represents an unknotted graph (i.e. a trivial link) under some over/under information. This fact is useful for the calculation of various link polynomials. See [1] for example. It is natural to ask whether or not this is true for planar graphs. In this paper we show that this is not true for planar graphs. Figure 1.1 is a regular projection of the regular octahedron graph. It is easily checked that all spatial graphs obtained from this projection contain at least one Hopf link. Therefore this projection cannot produce an unknotted graph under any over/under information of the double points.

A regular projection of a planar graph is called knotted if it cannot produce an unknotted graph under any over/under information of the double points. A planar graph is called trivializable if it has no knotted regular projections.

A minor of a graph $G$ is a graph obtained from $G$ by a finite sequence of

Received by the editors May 18, 1994.

1991 Mathematics Subject Classification. Primary 57M25; Secondary 57M15, 05 C10.

Key words and phrases. Knot, planar graph, regular projection. 


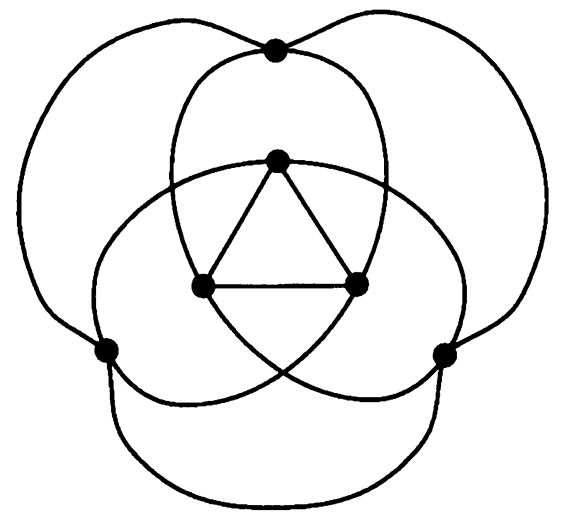

FIGURE 1.1

the following two operations: (1) edge contraction, (2) taking a subgraph. It is clear that a minor of a planar graph is planar. Moreover we will prove:

Proposition 1.1. A minor of a trivializable graph is trivializable.

We have seen that the regular octahedron graph is not trivializable. As a corollary we have the following main theorem of this paper.

Theorem 1.2. There are infinitely many planar graphs that are not trivializable.

In $\S 2$ we prove Proposition 1.1. In $\S 3$ we show that certain planar graphs are trivializable.

\section{Proof of Proposition 1.1}

Proof of Proposition 1.1. Suppose that $H$ is a subgraph of $G$ and $H$ is not trivializable. Then $H$ has a knotted regular projection $\widehat{H}$. Extend $\widehat{H}$ to any regular projection $\widehat{G}$ of $G . \widehat{G}$ is knotted because $\widehat{H}$ is knotted. Thus $G$ is not trivializable.

Next suppose that $H$ is obtained from $G$ by contracting an edge of $G$ and $H$ is not trivializable. Let $\widehat{H}$ be a knotted projection of $H$. Then it is clear that a regular projection obtained from $\widehat{H}$ as in Figure 2.1(a) is also knotted.

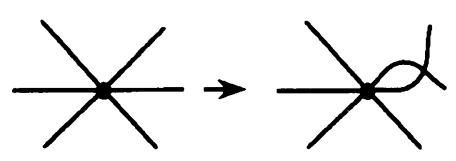

(a)

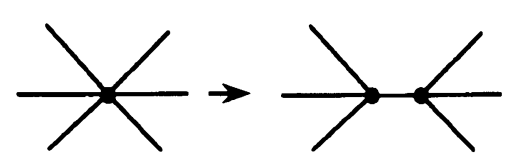

(b)

Figure 2.1 
After some applications of the operation of Figure 2.1(a) and an application of the operation of Figure 2.1(b) we have a regular projection $\widehat{G}$ of $G$. We now check that the operation of Figure 2.1(b) also preserves the knottedness of regular projection. Suppose that the right-hand side regular projection of Figure 2.1(b) can produce an unknotted graph under some over/under information. Then by the same over/under information the left-hand side regular projection produces a graph in $R^{3}$ that is obtained from an unknotted graph by contracting an edge in $R^{3}$. Therefore the graph is also unknotted. Thus the operation of Figure 2.1(b) preserves the knottedness. Therefore $\widehat{G}$ is knotted and $G$ is not trivializable.

\section{TRIVIALIZABLE GRAPHS}

In this section we show that certain planar graphs are trivializable.

A tree is a connected and simply connected graph. We construct a graph from a disjoint union of trees as follows. Let $T_{1}^{+}, T_{1}^{-}, T_{2}^{+}, T_{2}^{-}, \ldots, T_{n}^{+}$and $T_{n}^{-}$ be disjoint trees. Let $u^{+}$and $u^{-}$be vertices not on these trees. We join $u^{+}$ (resp. $u^{-}$) and each vertex of $T_{i}^{+}$(resp. $T_{i}^{-}$) by an edge for $i=1, \ldots, n$. Let $v_{i}^{+}$(resp. $\left.v_{i}^{-}\right)$be a vertex of $T_{i}^{+}\left(\right.$resp. $\left.T_{i}^{-}\right)$for $i=1, \ldots, n$. Then we identify $v_{i}^{+}$with $v_{i}^{-}$for $i=1, \ldots, n$. Let $G$ be the resultant graph. We call a graph that is homeomorphic to $G$ a bifocal. We will show that a bifocal is a planar graph. Moreover we have:

Theorem 3.1. A bifocal is trivializable.

Proof. Let $G$ be a bifocal constructed as above. Let $h: G \rightarrow R$ be a continuous function with the following properties:

(1) $h\left(v_{i}^{+}=v_{i}^{-}\right)=0 \quad(i=1, \ldots, n)$.

(2) $h(v)=d\left(v_{i}^{+}, v\right)$ if $v$ is a vertex of $T_{i}^{+}$where $d\left(v_{i}^{+}, v\right)$ is the number of edges of the path of $T_{i}^{+}$joining $v_{i}^{+}$and $v(i=1, \ldots, n)$.

(3) $h(v)=-d\left(v_{i}^{-}, v\right)$ if $v$ is a vertex of $T_{i}^{-}$where $d\left(v_{i}^{-}, v\right)$ is the number of edges of the path of $T_{i}^{-}$joining $v_{i}^{-}$and $v(i=1, \ldots, n)$.

(4) $h\left(u^{+}\right)=\max \left\{d\left(v_{i}^{+}, v\right) \mid v\right.$ is a vertex of $\left.T_{i}^{+}, i=1, \ldots, n\right\}+1$.

(5) $h\left(u^{-}\right)=-\max \left\{d\left(v_{i}^{-}, v\right) \mid v\right.$ is a vertex of $\left.T_{i}^{-}, i=1, \ldots, n\right\}-1$.

(6) The restriction $\left.h\right|_{e}$ is injective for each edge $e$ of $G$.

Let $\widehat{G}$ be a regular projection of $G$. Let $f: G \rightarrow R^{2}$ be a continuous map such that $\widehat{G}=f(G)$. We deform $f$ slightly if necessary so that the two points on a double point of $\widehat{G}$ take different values under $h$. Then we give over/under information coming from $h$ to the double points of $\widehat{G}$. Then the resultant embedding is ambient isotopic to the embedding $(f \times h): G \rightarrow R^{2} \times R^{1}$ defined by $(f \times h)(x)=(f(x), h(x))$. Let $p: R^{3} \rightarrow R^{2}$ be a projection defined by $p(x, y, z)=(x, z)$. We deform $f \times h$ slightly by an ambient isotopy if necessary so that $p \circ(f \times h)(G)$ is a regular projection. By shrinking the edges of $T_{i}^{+}$and $T_{i}^{-}$fixing $v_{i}^{+}=v_{i}^{-}$, we have that no double points are on $T_{i}^{+}$and $T_{i}^{-}$for $i=1, \ldots, n$. Then we eliminate the double points nearest to $u^{+}$and $u^{-}$respectively. Repeating this elimination we have a regular projection with no double points. Thus we have an unknotted embedding of a planar graph. See Figure 3.1. 

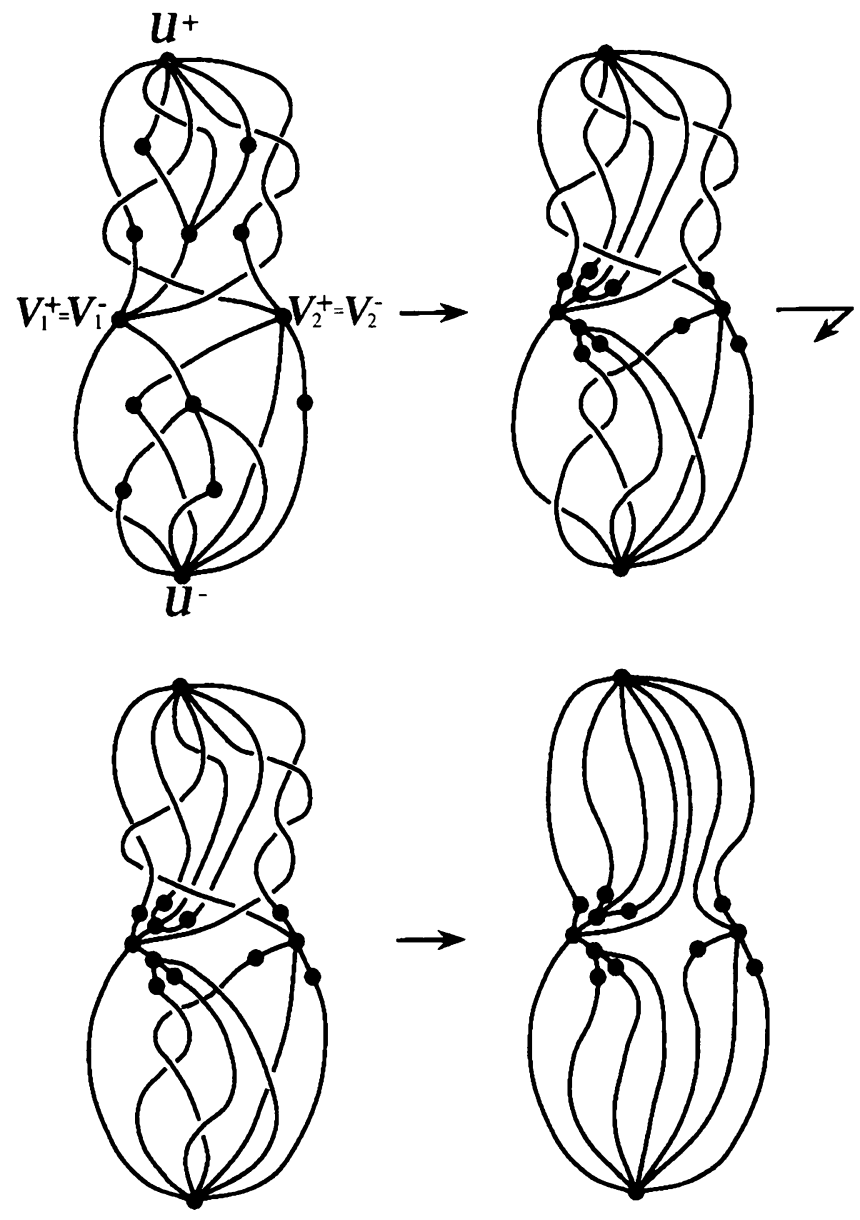

FIGURE 3.1

By Proposition 1.1 a minor of a bifocal is trivializable. Finally we prove the following proposition.

Proposition 3.2. (1) Let $G$ be a disjoint union of trivializable graphs $G_{1}$ and $G_{2}$. Then $G$ is trivializable.

(2) Let $G_{1}$ and $G_{2}$ be trivializable graphs and $v_{1}$ (resp. $v_{2}$ ) a vertex of $G_{1}$ (resp. $G_{2}$ ). Let $G$ be the graph obtained from $G_{1}$ and $G_{2}$ by the identification $v_{1}=v_{2}$. Then $G$ is trivializable.

Proof. (1) Let $\widehat{G}$ be a regular projection of $G$. Then $\widehat{G}=\widehat{G}_{1} \cup \widehat{G}_{2}$ where $\widehat{G}_{1}$ (resp. $\widehat{G}_{2}$ ) is a regular projection of $G_{1}$ (resp. $G_{2}$ ). We give over/under information to the double points of $\widehat{G}$ so that $\widehat{G}_{1}$ is always over $\widehat{G}_{2}, \widehat{G}_{1}$ represents an unknotted embedding of $G_{1}$ and $\widehat{G}_{2}$ represents an unknotted embedding of $G_{2}$. Then the resultant graph splits into two unknotted graphs in $R^{3}$. Thus the graph is unknotted.

(2) Let $\widehat{G}$ be a regular projection of $G$. We give over/under information to $\widehat{G}$ similarly to (1). Then the resultant graph is a union of two unknotted graphs. Moreover we may suppose that the intersection of the graph and $R^{2}\left(\subset R^{3}\right)$ is the vertex $v_{1}=v_{2}$. We choose a small ball $B^{+}$(resp. $\left.B^{-}\right)$in the upper 
(resp. lower) half-space that is tangent to $R^{2}$ at the vertex $v_{1}=v_{2}$. Then we shrink $G_{2}$ into $B^{-}$by an ambient isotopy that is fixed on the upper half-space. Since $G_{1}$ is unknotted, we can deform $G_{1}$ into $\partial B^{+}$by an ambient isotopy that preserves $B^{-}$. Then we deform $G_{2}$ into $\partial B^{-}$by an ambient isotopy that preserves $B^{+}$. Then the graph is clearly unknotted.

\section{REFERENCES}

1. P. Harpe, M. Kervaire, and C. Weber, On the Jones polynomial, Enseign. Math. 32 (1986), 271-335.

2. W. Mason, Homeomorphic continuous curves in 2-space are isotopic in 3-space, Trans. Amer. Math. Soc. 142 (1969), 269-290.

Department of Mathematics, College of Arts and Sciences, Tokyo Woman's Christian UNiversity, Zempukuji 2-6-1, SUginamiku, Tokyo, 167, JaPAN

E-mail address: taniyama@twcu.ac.jp 